\title{
RESÍDUOS SÓLIDOS DE SERVIÇOS DE SAÚDE: uma fotografia do comprometimento da equipe de enfermagema
}

\author{
Katsuy M eotti D OI ${ }^{b}$, G isela M aria Schebella Souto de M OU RA ${ }^{c}$
}

\section{RESUM 0}

0 presente trabal ho investigou os conhecimentos e atitudes dos profissionais da equipe de enfermagem do $\mathrm{H}$ ospital de Clínicas de Porto Alegre (HCPA), Rio Grande do Sul, em relação ao descarte dos resíduos sólidos dos serviços de saúde (RSSS). Realizou-se uma pesquisa de natureza exploratória descritiva, através de uma abordagem qual itativa. Os dados foram obtidos a partir de entrevistas semi-estruturadas aplicadas a 24 profissionais da equipe de enfermagem. Os dados foram categorizados através da análise de conteúdo, tendo sido identificadas quatro categorias: Significado da expressão resíduos sólidos de serviços de saúde; Separação dos resíduos sólidos dos serviços de saúde; Real ização do curso/ conhecimento e Profissionais que não real izam a separação. Os resultados ratificaram a importância de tratar com maior seriedade a questão apresentada, reforçando a necessidade do acesso às orientações adequadas.

D escritores: Resíduos de serviços de saúde. E quipe de enfermagem. Separ ação de resíduos sólidos.

\section{RESUMEN}

EI presente trabajo pretiende investigar los conocimientos y actitudes de los profesionales del equipo de enfermería de H ospital de Clínicas de P or to A legre (H CPA), R io G rande do Sul, B rasil, en relación al descarte de los residuos sólidos de los servicios de salud (RSSS). F ue realizada una investigación de naturaleza exploratoria descriptiva, a través de un abordaje cualitativo. $L$ os datos fueron obtenidos a partir de encuestas semi estructuradas aplicadas a 24 profesionales del equipo de enfermaje Se buscó la categorización de los datos a través del análisis de contenido. F ueron identificadas cuatro categorías: Significado de la palabra residuos sólidos de servicios de salud; Separación de los residuos sólidos de los servicios de salud; $R$ ealización del curso/ conocimiento y P rofesionales que no realizan la separación. Con los resultados, se ratificó la importancia de tratar con mayor seriedad la cuestión presentada, reforzando la necesidad del acceso a las orientaciones adecuadas.

D escriptores: R esiduos de hospitales. G rupo de enfer mería. Separación de residuos sólidos.

T ítulo: Residuos sólidos de servicios de salud: una fotografía del comprometimiento del equipo de enfermería.

\section{ABST RACT}

This work investigated know ledges and attitudes of nurses from the staff of $\mathrm{H}$ ospital deClínicas de P or to Alegre (H CPA), $\mathrm{R}$ io $\mathrm{G}$ rande do Sul, B razil, on solid healthcare waste (SH CW ). T his is an ex ploratory descriptive research with a qualitative approach. T he data were obtained from semi- structured interviews applied to 24 nurses from the staff. Content analysis was used to categorize the data. F our categories were identified: M eaning of the phrase solid healthcare waste; Separation of solid healthcare waste; R ealization of the course/ knowledge; and P rofessionals who do not separate. R esults confirmed the impor tance to treat this issue more seriously, emphasizing the need of access to appropriate guidance

Descriptors: M edical waste N ursing, team. Solid waste segregation.

$\boldsymbol{T}$ itle: $\mathrm{Solid}$ healthcare waste: a snapshot of the commitment of the nursing staff.

\footnotetext{
a Este artigo é um excerto do trabalho de conclusão do Curso de G raduação em E nfermagem apresentado em 2008 na Escola de Enfermagem da U niversidade Federal do Rio G rande do Sul (UFRGS).

b Enfermeira, Pós-G raduanda da E special ização em Enfermagem em UTI N eonatal do Centro Educacional São Camilo, Porto Alegre, Rio Grande do Sul, Brasil.

' D outora em Administração, Professora da Escola de Enfermagem da UF RGS, Porto Alegre, Rio Grande do Sul, Brasil.
} 


\section{INT RODUÇÃO}

0 crescimento tecnológico e industrial trouxe muitas consequências para a sociedade contemporânea, entre elas, 0 aumento da quantidade de lixo gerado pela população. Esses resíduos sólidos, quando não tratados de maneira correta e simplesmente despejados em locais inapropriados, acarretam muitos prejuízos a todo meio ambiente, afetando, assim, diretamente seu próprio gerador: 0 homem.

Entre as mais variadas formas de lixo, temos os resíduos sólidos dos serviços de saúde (RSSS), também denominado lixo hospitalar ou apenas resíduos dos serviços de saúde (RSS). Estes resíduos podem ser definidos como "rejeitos produzidos pelos mais diver sos estabelecimentos de saúde como: hospitais, clínicas veterinárias, farmácias, clinicas médicas e odontológicas, laboratórios entre outros"(1). Este tipo de lixo é subdivido em cinco grupos, o que se torna extremamente complexo a muitos profissionais da área da saúde que deveriam fazer sua separação.

$P$ ara que seja garantido o descarte correto desse lixo, existem normas que estão dispostas na Resolução $n^{\circ}$ 306/2004, da A gência N acional de Vigilância Sanitária (A NVISA)(2), e na de $n^{\circ} 358 / 2005$, do Consel ho $\mathrm{N}$ acional do $\mathrm{M}$ eio A mbiente (CONA$M A)^{(3)}$.

Pesquisa realizada anteriormente constatou que $58 \%$ dos profissionais não sabem o que são resíduos sólidos de saúde ou sabem mas não conseguem explicar a importância do manuseio e descarte adequado(4).

A penas $10 \%$ dos hospitais brasileiros dispõem de recursos profissionais capacitados para atuar na área de Higiene $\mathrm{H}$ ospitalar, e $90 \%$ não tem enfermeiros especializados nem verbas para investir em materiais e equipamentos que possam minimizar os problemas gerados pelo lixo hospitalar ${ }^{(5)}$.

O Plano de G erenciamento de Resíduos dos Serviços de Saúde é o documento que indica o caminho a ser percorrido pelo lixo hospitalar desde a destinação desses materiais até sua disposição final ${ }^{(6)}$. A importância desse gerenciamento é evidenciada a partir de estudos que comprovam os benefícios que este procedimento, quando realizado em acordo com a legislação vigente, trás a sociedade, ao meio ambiente e a própria entidade ${ }^{(7)}$.

No manual consta que o lixo hospitalar deve sofrer 10 processos: segregação, acondicionamento, identificação, armazenamento temporário, armazenamento exter no, coleta inter na, tratamento interno, coleta externa, tratamento externo e disposição final. Entre estes, a ação mais realizada pela equipe de enfermagem em questão é a segregação destes materiais, definida como "a separação dos resíduos no momento e local de sua geração, de acordo com as características físicas, químicas, biológicas, o seu estado físico e os riscos envolvidos"(8).

A separação do lixo hospitalar, no Hospital de Clínicas de Porto A legre (HCPA) - campo de estudo da presente investigação - baseia-se nos preceitos da Resolução 306/ 2004 da A NVISA (2).

Para a segregação do material, dispõe-se de recipientes revestidos internamente com sacos coloridos a fim de facilitar a visualização. 0 saco preto é destinado ao lixo comum, o saco verde ao lixo reciclável e o saco branco ao lixo contaminado. Conta, também, com recipientes de plástico devidamente rotulados para o descarte de substâncias químicas, como pilhas e sobras de medicamentos. Os materiais perfurocortantes são desprezados em caixas rígidas próprias para o uso, denominadas D escartex ${ }^{\circledR}$. São caixas de papelão identificadas com o símbolo de substância infectante, que possuem um pequeno orifício na tampa para o descarte e uma linha no bordo, a fim de orientar sua capacidade máxima de materiais.

A preocupação com os RSSS deve abranger tanto os profissionais de campo quanto aqueles indivíduos que estão em formação, isto é, os futuros profissionais. Estudo enfatiza a importância da abordagem dos Resíduos dos Serviços de Saúde nos cursos de graduação da área da saúde na promoção da conscientização dos sujeitos ${ }^{(8)}$.

E $m$ pesquisa realizada com trabal hadores da área médica e de enfermagem do HCPA constatou-se que os profissionais demonstraram conhecimento em relação aos materiais não específicos dos serviços de saúde. Este fato foi atribuído às campanhas realizadas e a divulgação pelos meios de comunicação(4).

O HCPA conta com um curso disponibilizado pelo Serviço de Higienização a todos os profissionais tanto das áreas administrativas quanto assistenciais. Ocorre às quartas-feiras e tem duração de 90 minutos, sendo uma semana no turno da manhã e na próxima no turno da tarde, facilitando 0 acesso dos profissionais. É dividido em duas etapas, onde, primeiramente, é incentivada à participação dos profissionais. Assim, cada um recebe 
um material simbolizando um tipo de lixo e deve desprezá-lo da maneira que considera correta. A pós cada descarte as ações são questionadas e discutidas. Num segundo momento é realizada uma aula expositiva, onde são esclarecidas as dúvidas.

A motivação para a realização da pesquisa surgiu após a observação de procedimentos realizados pela equipe de enfermagem durante o turno de trabalho, onde constatou que um número considerável destes profissionais não realizava o descarte adequado do lixo ger ado em suas atividades no hospital em estudo. Estes profissionais, embora instruídos a realizar uma boa assistência a seus pacientes e a colocar em prática técnicas aprendidas, não atentavam que a preocupação com 0 descarte dos RSSS também faz parte do cuidado.

Nesse contexto, o presente trabalho pretende investigar os conhecimentos e atitudes dos profissionais frente a essa problemática.

A credita-se que a realização da pesquisa contribuirá para uma maior reflexão por parte dos profissionais de enfermagem quanto ao descarte do lixo hospitalar.

\section{MÉT ODOS}

Este estudo caracteriza-se como uma pesquisa de natureza exploratória descritiva, através de uma abordagem qualitativa. 0 caráter exploratório possibilita maior clareza ao pesquisador quanto ao seu objeto de estudo, possibilitando um refinamento de suas ideias ou mesmo a identificação de novos pontos de vista. 0 principal objetivo da pesquisa descritiva é apresentar características de determinada população ou acontecimento, ou ainda a relação das variáveis descobertas. Este grupo compreende, também, a investigação da opinião de uma população(9).

0 estudo foi realizado em duas unidades de internação clínica e duas unidades de internação cirúrgica do H ospital de Clínicas de Porto Alegre (HCPA), Rio Grande do Sul.

A pesquisa teve como sujeitos os profissionais da equipe de enfermagem que realizam suas atividades de trabalho em unidades de internação clínica e cirúrgica, tendo sido entrevistados um enfermeiro e cinco auxiliares de enfermagem de cada unidade de internação, totalizando 24 profissionais. As entrevistas foram realizadas nos três turnos de trabal ho - manhã, tarde e noite - de acordo com a disponibilidade dos profissionais.
Os profissionais foram convidados a participar do estudo, sendo esclarecidos os objetivos da pesquisa e a não obrigatoriedade de adesão. $\mathrm{Na}$ coleta dos dados, foi realizada uma entrevista, onde foram questionados os motivos que os levam a não realizar a separação correta do lixo hospitalar, para aqueles que negarem tal prática. E, para aqueles que afirmam realizar a separação dos RSSS foram questionados os motivos que os levam a preocuparse com mais essa ação, dentre outras tantas que devem executar.

O bedecendo aos critérios de inclusão pré-estabelecidos, o profissional teve que pertencer à equipe de enfermagem do HCPA, realizar suas atividades nas unidades pré-estabelecidas, e estar há pelo menos seis meses em atividade profissional no HCPA.

0 critério de exclusão compreendeu os profissionais que estavam em período probatório, funcionários em licenças prolongadas e em férias, assim como aquel es que se recusaram a participar do estudo.

Para a realização da coleta de dados, foi realizada uma entrevista semi-estruturada na unidade de trabalho do profissional, em local reservado para garantir a privacidade das informações e do entrevistado. Cabe esclarecer que não havia relação hierárquica entre entrevistador e entrevistado.

A técnica de coleta de dados - entrevista - foi escol hida por se entender que em situações "face a face" pode-se considerar não só as respostas dadas pelo entrevistado, mas toda sua comunicação nãoverbal ${ }^{(9)}$. As perguntas eram pré-estabelecidas e, ao final, foi disponibilizado espaço para que o profissional fizesse as observações que considerava relevantes, contribuindo para a riqueza dos dados.

As respostas dadas pelos profissionais foram gravadas para garantir a veracidade do conteúdo e para que assim, no momento da separação das informações para categorização, não se perdesse nenhum detal he e não houvesse prejuízos na análise.

Os dados obtidos através das entrevistas foram analisados com base na técnica de análise de conteúdo, buscando a categorização dos mesmos. 0 método de categorização tem como primeiro objetivo promover uma classificação mais simples dos dados brutos. Os elementos foram, portanto, agrupados em razão de suas características comuns. A pós, foi definido o título de cada categoria(10).

A fim de garantir o anonimato dos sujeitos da pesquisa, durante os relatos e análises dos dados, 
identificou-se os integrantes através de uma codificação contendo a unidade de trabalho (4S, 7S, $7 \mathrm{~N}, 8 \mathrm{~S})$, um número correspondente à idade e a letra correspondente à categoria profissional (A eE).

0 Termo de Consentimento Livre e E sclarecido foi apresentado ao profissional a fim de ter seu consentimento em participar da entrevista. Foram seguidas as orientações da L ei de Direitos Autorais 9610/ $98^{(11)}$.

0 projeto foi submetido à avaliação e aprovado pela Comissão de Pesquisa da E scola de Enfermagem da Universidade Federal do Rio Grande do Sul (COM PESQ/ EEUFRGS) e pelo Comitê de Ética e Pesquisa do H ospital de Clínicas de Porto A legre (G PPG / H CPA), sendo aprovado sob o protocolo $\mathrm{n}=08-361^{(12)}$.

\section{RESULT ADOS}

A entrevista foi realizada com 24 profissionais da equipe de enfer magem do H ospital de Clínicas de Porto Alegre, sendo constituída a amostra de 20 auxiliares de enfermagem (83\%) e 4 enfermeiros (17\%). Quanto ao sexo, 22 (92\%) eram do sexo feminino. A faixa etária predominante foi de 36-45 anos (67\%), os restantes situaram-se na faixa etária de 20-35 anos (33\%). Quanto ao tempo de trabalho, o grupo de participantes dividiu-se em 18 entrevistados (75\%) com menos de 10 anos de atividade na instituição e 6 (25\%) com mais de 10 anos.

A predominância do sexo feminino na amostra estudada é semelhante a outras situações descritas na literatura que destacam o maior quantitativo feminino na profissão de enfermagem, relacionando este aos valores culturais, em que a muIher tem mais desenvolvido o dom de cuidar ${ }^{(13)}$. Outro autor relaciona este fato à questão da inferioridade feminina, muito defendida no início do século $X X$, onde vêem a mulher como um ser com capacidades submissas ao homem, aqui no caso representado pelo profissional médico ${ }^{(14)}$.

As entrevistas seguiram um roteiro pré-estabelecido, conforme descrito no método. Foram consideradas também, na análise, as manifestações não previstas no roteiro, pois se entendeu como informações relevantes para a pesquisa na construção das categorias.

A análise de conteúdo dos relatos evidenciou quatro categorias descritas a seguir.

\section{Categoria 1: Significado da expressão resíduos sólidos de serviços de saúde}

Quando questionados acerca do significado da expressão resíduos sólidos de serviços de saúde, apenas 13 profissionais afirmaram saber seu significado, sendo que destes, apenas nove definiram adequadamente, tendo a pal avra lixo sido mencionada em sete das respostas.

0 nosso lixo (4S-42A).

Todo lixo que sobra (7S-49A).

\section{Categoria 2: Separação dos resíduos sólidos dos serviços de saúde}

E $m$ relação à separação desses resíduos, 22 dos entrevistados asseguraram realizar a separação dur ante sua rotina de trabal ho. Porém, quando apurados os critérios utilizados por eles, verificouse que apenas nove profissionais desempenham tal ação de maneira adequada, de acordo com as normas preconizadas pelo HCPA.

A falha mais observada foi a colocação da manta esterilizada pelo Centro de $M$ ateriais (CM E) do HCPA nos lixos branco e verde (8), sendo o correto o seu descarte em lixo comum, isto é, o lixo preto.

[ ...] A manta eu ponho no branco, boto tudo junto, pois no curativo quando eu boto a pinça no campo eu considero ele contaminado eassim a manta eu considero também contaminada (4S-45E).

[...] A manta do curativo é lixo reciclável (7S46A).

Também se constatou o descarte do equipo de soro de maneira incorreta em quatro casos, sendo três em lixo verde e um em lixo preto, os quais deveriam ser desprezados em lixo contaminado.

[ ...] 0 equipo eu boto no preto, não sei se tá correto [...] $(7 \mathrm{~N}-42 \mathrm{~A})$.

[ ...] E quipo ponho no verde, de plástico, a não ser que seja de quimio, que ai vai para o material de quimio [ ...] (4S-45E).

Observaram-se ainda, um caso de luva e outro de frasco de soro em saco preto, necessitando 
serem desprezados em lixo contaminado e verde, respectivamente.

Lixo preto a gente pode botar papel, lixo alimentar, luva, toca, máscara [ ...] $(7 \mathrm{~N}-39 \mathrm{E})$.

[ ...] O frasco do soro quando limpo eu boto no preto, se não eu boto no branco $(7 \mathrm{~N}-42 \mathrm{~A})$.

Constatou-se, inclusive, um profissional que coloca gazes não utilizadas e outro que descarta as fraldas em lixo branco, sendo estes materiais desprezados adequadamente em lixo comum.

$\mathrm{N}$ o branco vão as gazes sujas e gaz es que são abertas $\mathrm{e}$ não são utilizadas [ ...] (8S-34A).

A gentetem todos os lix os separadinhos, ea gente procura colocar o que é fralda no saco branco [ ...] (7N $42 \mathrm{~A})$.

Outro caso de seringa de medicação sendo depositada no Descartex ${ }^{\circledR}$, caixa de papelão própria para seringas contaminados com a presença da agulha, sendo o correto seu descarte em lixo branco.

[ ...] O D escartex ${ }^{\circledR}$ é onde vão as seringas e agulhas [...] (4S-31A).

Ainda, um profissional afirmou desprezar restos de alimentos em saco verde, recipiente destinado a materiais recicláveis. De acordo com as normas restos de al imentos devem ser desprezados em recipiente destinado ao lixo comum, próprio para resíduos que não contém material biológico.

L ixo seco a gente coloca papel, restos de comida (7N34A).

\section{Categoria 3: Realização do curso/ conhecimento}

Dos seis profissionais que relataram terem participado do curso de reciclagem ofer ecido pelo Serviço de Higienização do hospital, quatro separam o lixo corretamente.

E assim eu faço a divisão conforme com o que eu aprendi em cursos de reciclagem. F icou bem claro, e eles estão sempre atualizando. $\mathrm{N}$ ão acontece ainda de ficar na dúvida, por enquanto não (8S-32A).

\section{Categoria 4: Profissionais que não realizam a separação}

Os dois profissionais que confessaram não descartar sempre o lixo no local adequado usaram 0 argumento da falta de tempo para justificar suas ações. U m deles acrescentou, ainda, que o número de profissionais da unidade é inadequado, não sendo possível realizar a assistência ao paciente e preocupar-se também com o descarte do material utilizado. A fir mou que muitas vezes precisa optar entre uma assistência ou o descarte adequado, sendo a prioridade, então, o paciente.

Às vezes não dá. Por ex emplo, eu sei que as luvas não são nos lixos dos quartos, mas a gentenão pode toda a vez que a gente vai trocar uma luva sair de lá e pegar um saco branco para colocar as luvas. Teve um paciente que tava trocando a fralda, sujei a luva, eu tive que trocar a luva, lavar a mão e não tinha como atravessar todo o corredor com uma luva na mão (7S49A).

\section{DISCUSSÃO}

Os resultados apresentados comprovam que a denominação "Resíduos Sólidos dos Serviços de Saúde" não era do conhecimento da maioria dos profissionais por ser uma expressão raramente utilizada, sendo mais comum a utilização da denominação lixo hospitalar. A palavra "sólido" da expressão dificultou o entendimento por parte dos entrevistados, causando estranhamento em muitos deles quando explicado seu significado.

Constatou-se que, apesar dos profissionais afirmarem realizar a separação do lixo hospitalar, a maioria destes desconhece as normas, realizando a ação de maneira inadequada. Podemos sugerir que esta prática seja fruto da ação inconsciente, porém, os prejuízos que esta causa são igualmente danosos quando comparados a um profissional que realiza a prática conscientemente. $\mathrm{E} m$ recente estudo realizado em Instituição Federal de E nsino Superior comprovou-se que as orientações referentes ao assunto em questão deveriam iniciar durante a formação dos profissionais de saúde e enfatizado em mais de um momento durante a graduação, necessidade esta exposta por uma aluna de um curso da área da saúde ${ }^{(15)}$.

A lém deste fato, a equipe de enfermagem, devido à tendência de assumir o papel de administradora da instituição, possui um maior envolvimen- 
to no manejo dos RSSS. Porém, em alguns casos, evidencia-se o descomprometimento dos próprios formadores dos futuros profissionais, isto é, os professores, em relação à segregação desses resíduos, o que dificulta o desenvolvimento da prática adequada ${ }^{(16)}$. A pouca valorização demonstrada pelo professor e a ausência de um modelo a ser imitado pelo aluno origina uma lacuna na formação profissional que se refletirá, numa situação futura, em descaso com o descarte.

A colocação da manta do curativo esterilizada pelo CME em lixos verdes pode ser relacionada à constituição do material, tecido de aspecto semeIhante ao antigo papel que revestia os primeiros pacotes. Já a colocação em lixo branco é explicada pelos profissionais que a considera contaminada uma vez que entra em contato com as pinças contaminadas utilizadas na real ização do curativo.

0 descarte inadequado do equipo de soro em lixo verde e das luvas em lixo preto está relacionado ao fato do lixo contaminado ser considerado apenas aquele com secreções biológicas visíveis. Já o equipo e o frasco de soro desprezados em lixo preto, o lixo comum, pode estar relacionado à presença de líquido, o soro fisiológico.

As fraldas foram consideradas contaminadas por conterem eliminações biológicas, prática até pouco tempo tida como correta pela própria instituição em questão.

As seringas são desprezadas no Descartex ${ }^{\circledR}$ por ser o local destinado à colocação de materiais perfuro-cortantes, como as agulhas, evidenciando a compreensão das instruções da segregação de maneira equivocada. Cabe esclarecer que a seringa, quando acoplada à agulha, deve ser desprezada no D escartex ${ }^{\circledR}$, mas quando se encontra sem a presença da agulha, deve ser descartada no lixo branco.

N otou-se que a maior adesão à prática correta esteve relacionada aos profissionais que realizaram o curso proposto pela instituição. Podese, assim, supor que o curso alcançou seu objetivo, proporcionando a informação adequada e incentivando sua prática.

A penas dois profissionais manifestaram não realizar sempre a separação correta dos resíduos, porém, não podemos comprovar que os demais realmente desempenham tal ação rotineiramente, como foi afirmado pelos mesmos. As respostas afirmativas podem estar relacionadas ao medo da repressão por parte da instituição.
0 argumento utilizado por um dos entrevistados a fim de justificar sua prática inadequada foi relacionado somente às falhas institucionais, explicado através da escassez de recursos humanos, não sendo visto pelo profissional como uma responsabilidade sua como cidadão. A outra entrevistada refere-se à área física como um empecilho na separação.

\section{CONSIDERAÇÕES FINAIS}

A pós a realização da coleta dos dados e anál ise das entrevistas, constatou-se que a abordagem referente aos resíduos sólidos de ser viços de saúde ainda é insuficiente, causando, assim, muitos prejuízos no âmbito institucional e coletivo.

D urante as entrevistas, evidenciou-se o interesse de alguns profissionais em contribuir para a realização adequada do processo, porém, identificaram-se fatores que agiram como limitantes.

A falta de orientação adequada esteve presente em muitos relatos, onde apesar da maioria dos profissionais (22) ter afirmado realizar a separação dos RSSS, quando questionado quanto aos critérios utilizados, relataram ações que não condiziam com normas utilizadas como referência pela instituição. Alguns, inclusive, aproveitaram o momento da entrevista para esclarecer dúvidas.

$\mathrm{N}$ otou-se, também, a ausência de interesse por parte de profissionais que utilizaram argumentos como falta de tempo para justificar a não realização do curso proporcionado pelo hospital.

Pode estar relacionado a este fato o não conhecimento destes profissionais em relação aos impactos que suas ações inadequadas causam a nível populacional, refletindo em aumento de custos e prejuízo ambiental. Observou-se que estes não vêem a separação adequada como responsabilidade tão importante quanto 0 atendimento prestado ao paciente.

Os entrevistados que manifestaram não realizar sempre a separação do lixo, utilizaram argumentos para explicar suas ações. 0 primeiro deles apontou a escassez de recursos humanos para a realização da assistência como o principal fator limitante no processo. Outro profissional relatou a área física como principal empecilho no descarte adequado.

A credita-se que o objetivo do estudo, qual seja, 0 de investigar os conhecimentos e atitudes dos profissionais frente ao descarte do RSSS tenha sido 
atingido em sua totalidade. Os achados apontam para a premência de inter venção disparada pelo hospital, mediada por estratégias que levem a reflexão das práticas atuais por parte dos profissionais e, concomitantemente, comprometendo a equipe com a busca e a adesão às soluções capazes de desenhar um novo cenário institucional. Vislumbra-se, ainda, o imperativo de orientação e supervisão contínuas como abordagens educativas junto à equipe de enfermagem para favorecer a adoção de atitudes desejáveis com o descarte dos RSSS.

Ao tér mino do presente estudo ressal ta-se a importância da discussão do tema em foco e a necessidade de novos estudos para aprofundamento do mesmo, buscando alternativas de soluções para melhorar o processo de descarte dos resíduos sólidos dos serviços de saúde, através de ações que contribuam para formação de sujeitos cidadãos.

\section{REFERÊNCIAS}

1 Nóbrega CC, Paes RF C, N eto JPF, Lima J D, Ruberg C. Diagnóstico dos resíduos sólidos de serviços de saúde provenientes de hospitais e clínicas médicas do município de João Pessoa - PB. In: A nais do 6o Simpósio Í talo B rasileiro de E ngenharia Sanitária e A mbiental; 2002 set 1-5; Vitória, Brasil. Vitória: ABE S; 2002. p. $1-9$.

2 A gencia N acional de Vigilância Sanitária (BR ). RDC no 306, de 7 de dezembro de 2004: dispõe sobre o Regulamento T écnico para o gerenciamento de resíduos de serviços de saúde. Brasília (D F ); 2004.

3 Conselho $\mathrm{N}$ acional do M eio Ambiente (BR). Resolução no 358, de 29 de abril de 2005: dispõe sobre 0 tratamento e a disposição final dos resíduos dos serviços de saúde e dá outr as providências. Brasília (DF); 2005.

4 N aime R, Ramal ho AH P, N aime IS. Avaliação do sistema de gestão dos resíduos sólidos do H ospital de Clínicas de Porto Alegre. Espaç Saúde. 2007;9(1):117.

5 Belei RA, T avares M S, Paiva NS. Lixo e serviços de saúde: uma revisão. E spaç Saúde. 1999;1(1):1-22.

\section{Endereço da autora / Dirección del autor / Author's address:}

Katsuy M eotti D oi

Rua Carlos Ferreira, 45, T eresópol is

91720-040, Porto Alegre, RS

E-mail: katinhamd@yahoo.com.br
6 Jácomo M VJ. M anual de gerenciamento de resíduos de serviços de saúde [ I nternet] . Goiás: Superintendência de Vigilância Sanitária e A mbiental; 2004 [ citado 2008 jun 05] . D isponível em: http:/ / www. visa. goias.gov.br/ arquivos/ manual_PG RSS.pdf.

7 Camargo M E, M otta EV, Lunelli M O, Severon EA. Resíduos Sólidos de Serviços de Saúde: um estudo sobre o gerenciamento. Sci Plena. 2009;5(7):1-14.

8 Corrêa L B, L unardi VL, D e Conto SM . 0 processo de formação em saúde: o saber resíduos sólidos de serviços de saúde em vivências práticas. Rev Bras E nferm. 2007;30(1):21-5.

9 Gil AC. Como elaborar projetos de pesquisa. 4a ed. São Paulo: A tlas; 2002.

10 Bardin L. A nálise de conteúdo. 3ạ ed. Lisboa: E dições 70; 2004.

11 M inistério da Justiça (BR). Lei o 9610, de 19 de fevereiro de 1998: altera, atualiza e consolida a legislação sobre direitos autorais e dá outras providências. Brasília (DF); 1998.

12 D oi KM . Resíduos de Serviços de Saúde: percepção dos profissionais de saúde quanto o seu descarte [ monografia] . Por to A legre: E scola de Enfermagem, U niversidade Federal do R io G rande do Sul; 2008.

13 A ssunção LA , Costa CS, Lourença HF. M itos na enfer magem: mulher, trabalho e gênero. In: A nais do 1 - Congresso I nter nacional sobre a M ulher, G ênero eRelações de T rabal ho; 2005 maio 5-7; G oiânia, Brasil. G oiânia: Procuradoria Regional do T rabalho da 18a Região; 2005. p. 1-3.

14 Coel ho EAC. G ênero, saúde e enfer magem. Rev Bras de Enferm [ Internet] . 2005 [ citado 2008 set 07]; 58(3):345-8. Disponível em: http:/ / www.scielo.br/ $\mathrm{pdf} /$ reben/ v58n3/ a18v58n3.pdf.

15 Corrêa LB, L unardi VL, Santos SSC. Construção do saber sobre resíduos sólidos de serviços de saúde na formação em saúde. Rev G aúcha E nferm. 2008;29 (4):557-64.

16 Corrêa L B, Lunardi VL, Conto SM . 0 processo de for mação em saúde: o saber resíduos sólidos de serviços de saúde (RSSS) em vivências práticas. Rev Bras Enferm. 2007;60(1):21-5.

Recebido em: 13/ 07/ 2010

A provado em: 01/ 06/ 2011 